\title{
USING CUCKOO ALgORITHM FOR ESTIMATING TwO GLSD PARAMETERS AND COMPARING IT WITH OTHER ALGORITHMS
}

\author{
Jane Jaleel Stephan, Haitham Sabah Hasan and Alaa Hamza Omran \\ University of Information Technology \& Communications, Iraq
}

\begin{abstract}
This study introduces and compares different methods for estimating the two parameters of generalized logarithmic series distribution. These methods are the cuckoo search optimization, maximum likelihood estimation, and method of moments algorithms. All the required derivations and basic steps of each algorithm are explained. The applications for these algorithms are implemented through simulations using different sample sizes $(n=15,25,50,100)$. Results are compared using the statistical measure mean square error.
\end{abstract}

\section{KEYWORDS}

Cuckoo search optimization (CSO) algorithm, maximum likelihood estimation (MLE) algorithm, method of moments (MOM) algorithm, mean square error (MSE).

\section{INTRODUCTION}

The process of modifying a system to present several new features that corporate in enhancing the system and work more efficiently is known as optimization process. Also the Optimization process can be defined as the process of finding alternative solution to increase the performance of the system under specific constraints such as increasing the desired parameters and decrease the undesired parameters in the system which has a problem to solve it [1]. The increasing means trying to get additional good results without additional cost such as the optimization which occurs on computer or any android phone will results in increasing the speed of processing which makes them run faster with less memory requirements. There are many algorithms in solving optimization problems such as cukoo search algorithm which introduced for the first time by Yang and Deb [2]. Many researchers work on testing this algorithm on some benchmark functions and compare the results with other algorithms like PSO, GA; the obtained results show that the cukoo algorithm is better than the others. One of the popular met heuristic, combinatorial search optimization techniques is ACO (Ant Colony Optimization) which is developed from natural ant behavior ACO was used along with Rough Sets and Fuzzy Rough Sets in feature selection in [3], [4], [5] also it is used for optimizing of firewall rules in [6].

Today the Cuckoo search algorithm became as the one of the most optimization algorithm which used in every domain like scheduling planning, forecasting, image processing, feature selection and engineering optimization [7]. This paper presents a Comparing of the Cuckoo Algorithm with Other Algorithms for Estimating Two GLSD Parameters. Some important functions are defined as follows:

The discrete random variable (x) exhibits the generalized logarithmic series distribution (GLSD) with two parameters ( $\alpha$ and $\beta$ ), where $(\alpha)$ is a scale parameter and $(\beta)$ is a shape parameter. Let 
International Journal of Computer Science \& Information Technology (IJCSIT) Vol 9, No 5, October 2017

$$
\theta=-\frac{1}{\log (1-\alpha)}
$$

Where $\Theta$ is a function from $\alpha$. The positive matrix factorization (p.m.f.) of GLSD is defined by Eq. (1) as follows:

$$
p(X=x)=\frac{\theta \Gamma(\beta x) \alpha^{2}(1-\alpha)^{\beta x-x}}{x \mid \Gamma(\beta x-x+1)}
$$

Where $x=1,2, \ldots, \infty, \beta \geq 1,0 \leq \alpha \leq 1 / \beta, 0<\theta<1$.

The distribution in Eq. (1) depends on the zero-truncated generalized negative binomial defined by Eq. (2):

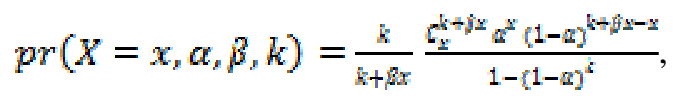

Where $x=1,2, \ldots, \infty, \quad k>0 \quad 1 \leq \beta \leq \frac{1}{g}$.

When limit $(\mathrm{k} \rightarrow 0)$ is considered for Eq. (2), we obtain the studied distribution in Eq. (1).

The mean of GLSD is defined in Eq. (1), and the variance obtained from the general formula of the $\left(\mathrm{k}^{\text {th }}\right)$ moments about the mean is as follows:

$$
\begin{aligned}
\mu_{k} & =E(x-\mu)^{k}, \quad \theta=[\ln (1 \quad \alpha)]^{-1}, \\
\mu_{1} & =E(x)=\frac{\alpha \theta}{(1-\alpha \beta)}, \\
\sigma^{2} & =\mu_{2}-\mu_{1}^{2}=\frac{(1-\alpha)\left[2 \alpha \beta-\alpha-\alpha^{2} \beta\right]}{(1-\alpha \beta)^{2}} .
\end{aligned}
$$

\section{Estimating Parameters}

We apply different methods for the p.m.f. parameters in Eq. (1).

\subsection{Maximum Likelihood Estimation (MLE)}

The Maximum Likelihood Estimation (MLE) [8], [9] that corresponds to Eq. (1) is given by:

$$
\begin{aligned}
& L\left(x_{i}, x_{i} \beta\right)-\prod_{i=1}^{n} p\left(X_{i}-x_{i}\right) \\
& \log L= \\
& n \log \theta+n \bar{x} \log \alpha+\sum_{x=1}^{k} \sum_{j=1}^{x-1} f(x) \log (\beta x- \\
& i)+n \bar{x}(\beta-1) \log (1-\alpha)-\sum_{i=1}^{\infty} f\left(x_{i}\right) \log x_{i} ! \\
& 2 \log L={ }^{n} \bar{x} \mid \begin{array}{c}
n \bar{x}(\beta-1) \quad n \theta \\
2 \alpha
\end{array} \quad 0, \\
& \text { And } \frac{\partial \log L}{\partial \beta} \Rightarrow-\frac{n \bar{x}}{\theta}+\sum_{x=1}^{k} \sum_{j=1}^{x-1}\left[\frac{x f(x)}{(\beta x-j)}\right] .
\end{aligned}
$$

This equation derives:

From $\frac{\partial \log L}{\partial \alpha}=0$, we obtain $\hat{\alpha}_{M L E}$.

$$
\hat{f}_{M L E}=\frac{1}{a}-\frac{a}{x}
$$


International Journal of Computer Science \& Information Technology (IJCSIT) Vol 9, No 5, October 2017

\subsection{Method of Moments (MOM) Estimator for GLSD Parameters}

Method of Moments (MOM) Estimator for GLSD Parameters [10], these estimators $\left(\hat{\alpha}_{\text {mom }}, \ddot{\beta}_{\text {mom }}\right)$ are obtained by solving the following:

$$
\begin{gathered}
\mu_{r}^{r}=m_{r}^{r}, \\
m_{r}^{\prime}=\sum_{x=1}^{\infty} x^{r} p(X=x) .
\end{gathered}
$$

When $r=1$,

$$
\begin{gathered}
m_{1}^{r}=\sum_{x=1}^{\infty} \frac{x \theta \Gamma(\beta N) \alpha^{n}(1-\alpha)^{\beta N-x}}{x ! \Gamma(\beta x-x+1)}, \\
m_{1}^{r}=\bar{x}=\frac{1-\alpha}{(1-\alpha \beta)^{2}} .
\end{gathered}
$$

When $r=2$,

$$
\begin{gathered}
m_{2}^{l}=\sum_{x=1}^{\infty} \frac{x^{2} \theta \Gamma(\beta x) \alpha^{n}(1-\alpha)^{\beta *-x}}{x+\operatorname{T}(\beta x-x+1)}, \\
m_{2}^{\prime}=\theta(1-\alpha \beta)^{-3} \alpha(1-\alpha) .
\end{gathered}
$$

Given that:

and

$$
\bar{x}^{2}=\frac{(1-\alpha)^{2}}{(1-\alpha \beta)^{4}}
$$

then,

$$
\sigma^{2}=E\left(x^{2}\right)-[E(x)]^{2}
$$

We have

$$
s^{2}=m_{2}^{\prime}-\left(m_{1}^{\prime}\right)^{2}=\frac{(1-\alpha)\left(2 \alpha \beta-\alpha-\alpha^{2} \beta\right)}{(1-\alpha \beta)^{4}}
$$

$$
\begin{gathered}
m_{1}^{r}=\theta(1-\alpha \beta)^{-1} \alpha \\
m_{2}^{i}=\theta(1-\alpha \beta)^{-3} \alpha(1-\alpha),
\end{gathered}
$$

and

$$
S^{2}=m_{2}^{\prime}-\left(m_{1}^{\prime}\right)^{2}
$$

which is simplified as follows:

$$
S^{2}=\frac{(1-\alpha)\left(2 \alpha \beta-\alpha-\alpha^{2} \beta\right)}{(1-\alpha \beta)^{4}} .
$$

We also obtain the following:

Then,

$$
\bar{x}^{2}=\frac{(1-\alpha)^{2}}{(1-\alpha \beta)^{2}} .
$$

$$
\frac{s^{2}}{x^{2}}=\frac{\left(2 x \beta-\alpha-\alpha^{2} \beta\right)}{(1-\alpha)} .
$$


We derive the first three non-central moments obtained from $\left[m_{r}^{t}=E\left(x^{r}\right)\right]$.

Then,

$$
\begin{aligned}
& m_{1}^{r}-\theta(1 \quad \alpha \beta)^{-1} a, \\
& m_{2}^{\prime}=\theta(1-\alpha \beta)^{-3} \alpha(1-\alpha), \\
& m_{3}^{r}=\frac{\theta \alpha(1-\alpha)\left(1-2 \alpha+2 \alpha \beta-\alpha^{-1} \beta\right)}{(1-\alpha \beta)^{5}} .
\end{aligned}
$$

Given that:

$$
m_{r}^{\prime}=\frac{\sum_{x_{1}}{ }^{n}}{n}
$$

We obtain the following based on the preceding relation:

$$
\begin{gathered}
\frac{\sum x_{i}^{3}}{\sum x_{i}^{2}}=\frac{\left[(1-\alpha)+\frac{s^{2}}{\bar{x}^{2}}(1-\alpha)\right]}{(1-\alpha \beta)^{2}}, \\
\frac{\sum x_{i}{ }^{3}}{\sum_{x} x_{i}^{2}}=\frac{(1-\alpha)\left(1+\frac{s^{2}}{z^{2}}\right)}{(1-\alpha \beta)^{2}} .
\end{gathered}
$$

Eq. (14) can be simplified into

$$
\frac{\bar{x}^{2} \sum x_{i}^{2}}{\sum x_{i}^{2}}\left(1+\frac{s^{2}}{x^{2}}\right)=\frac{\alpha^{2} e^{2}}{(1-\alpha)}
$$

Given that

Eq. (15) can be written as follows:

$$
\theta=-\frac{1}{\log (1-\alpha)}
$$

$$
\frac{x^{2} \sum x_{i}^{2}}{\sum x_{i}^{2}}\left(1+\frac{s^{2}}{x^{2}}\right)=\frac{a^{2} \log (1-x)^{2}}{(1-\alpha)},
$$

which is an implicit function that can be solved numerically to determine ( $\hat{\alpha}_{\text {mom }}$ ) based on observation. We then obtain $\left(\hat{\beta}_{\text {mom }}\right)$ by using $\left(\hat{\alpha}_{\text {mom }}\right)$ and solving Eq. (11).

\subsection{Cuckoo Search Optimization (CSO) Algorithm}

This algorithm is based on the breeding behaviour of the cuckoo bird. It has three basic optimization rules [11], [12].

1. Each bird lays one egg at a time, and each egg is placed randomly in a selected nest.

2. The best nest with the highest fitness value will be carried over to the next generation.

3. The number of available host nests is fixed. $\left(p_{\alpha x} \in[0,1]\right)$ represents the probability of the host bird discovering egg/s in its nest. The host bird can either throw away the egg/s or abandon the nest to build a new one [13][14].

The latter scenario can be regarded as the new best solution.

Let: 
$X_{i}^{(t)}$ Be the nest where the cuckoo bird initially lives, and

$X_{i}^{(t+1)}$ Be the new nest with the highest fitness value.

When random walk is used, the performance of the cuckoo (i) that applies levy flight is expressed as [5, 7]:

$$
X_{i}^{(t+1)} X_{i}^{(t)}+S \text { levy } \lambda
$$

Levy flight was first introduced by the French mathematician Paul Pierre.

$$
5 \sim \operatorname{Nomal}\left(\mu_{10}, \sigma^{2}=1\right)
$$

The probability $\left(p_{\alpha} \in[0,1]\right)$ indicates that the egg/s in the nest may be from another bird, and thus, the cuckoo bird may leave this host nest and build another one. The $n$ hosts may be changed to new hosts with random positions (probability $p_{u s}$ of change). Thus, the objective function belongs to the maximization type and the objective must be fitted into this type. The most important algorithm that can be applied is one used to solve nonlinear equation problems or one used in neural networks because these objects allow the algorithm to be transformed from state to state to reach the optimal solution. Given that GLSD has two parameters $(\theta$ and $\beta)$, then the algorithm implements the following steps.

Each bird lays one egg at a time in a randomly selected nest. The number of selected nests is equal to the number of parameters to be estimated. The number of nests is determined from the following equation:

Number of nests $=L B+(U B-\mathrm{LB}) \times$ random number $(0,1)$.

Let $X_{i}^{(\tau)}$ be the nest where the cuckoo bird initially lives.

$X_{i}^{(t+1)}$ is the new nest with the highest fitness value.

$$
X_{i}^{(t+1)} X_{i}^{(\omega)}+S t^{-\lambda}
$$

Each nest contains the parameters to be estimated, and the number of nests is also determined based on these parameters.

Step (1):

Number of nests $=L B+(U B-L B) \times$ random number $(0,1)$

Step (2):

The objective function for each nest is calculated as follows:

$$
0=\sum_{i=1}^{m}\left[F\left(x_{(i)}, \hat{\theta}, \hat{\beta}\right)-\frac{n x_{(\omega)}}{n}\right]
$$


Step (3):

The best values of the parameters determine the best nest with respect to the eggs.

Step (4):

The repetition begins. Let

$X_{i}^{(t+1]}$ be the nest in which the cuckoo bird initially lives, and

$X_{i}^{(t+1)} X_{i}^{(t)}+S t^{-\lambda}$ be the new nest with the highest fitness value.

Step (5):

A new nest is generated for the cuckoo from $k$, as follows:

$U=\operatorname{rand}(1,2) k$,

$$
k=\left[\Gamma(1+\beta) \frac{\sin \left(\pi_{i n}^{\beta}\right)}{\mathrm{T}\left(\frac{1+\beta}{\Omega}\right) \beta 2 \frac{\beta-1}{\Omega}}\right]^{1 / \beta},
$$

$L=\operatorname{rand}(1,2)$,

step: $\frac{U}{\left[\left.L\right|^{1 / 6}\right.}$

$$
\begin{aligned}
& \text { step size }=(0.01) \text { step }(\text { nest }- \text { best }), \\
& \text { new nest }=n \text { set }+ \text { step size } \times \text { rand }(1,2) \text {. }
\end{aligned}
$$

Step (6):

The objective function for each new nest is computed.

Step (7):

The solution is continued until the stopping rule ends with the total frequency. The best solution determined is then printed.

The CSO algorithm, which represents a meta-heuristic algorithm, is adopted to estimate $\left(\theta^{\wedge}, \beta\right)$. Then, $\left(\theta^{\wedge}\right)$ provides the estimate of $(\alpha)$. More details on this algorithm are explained in detail in [15].

\section{SimULATION}

The three estimators of ( $\alpha$ and $\beta$ ), i.e., the CSO, MLE, and MOM algorithms, are compared through MATLAB: A11 program. Different sample sizes $(n=15,25,50,100)$ are considered, and the results are compared using the statistical measure mean square error (MSE) and run of each experiment $(R=1000)$. 
International Journal of Computer Science \& Information Technology (IJCSIT) Vol 9, No 5, October 2017

TABLE 1: Comparison of the Different Estimators When $\beta=1.5$ and $\alpha=0.3$

\begin{tabular}{|c|c|c|c|c|c|}
\hline$n$ & Method & $\beta=1.5$ & $\alpha=0.3$ & Kurtosis & Skewness \\
\hline \multirow{7}{*}{15} & $\mathrm{mle}$ & 1.0512 & 0.3056 & 1 & 0 \\
\hline & mse_mle & 0.6889 & 0.0019 & & \\
\hline & mom & 1.0961 & 0.7347 & & \\
\hline & mse_mom & 0.8226 & 0.1897 & & \\
\hline & cuck 00 & 1.3836 & 0.3010 & & \\
\hline & mse_cucko 00 & 0.0313 & 0.0014 & & \\
\hline & best & cuck 00 & cuck 00 & & \\
\hline & & & & & \\
\hline \multirow{7}{*}{25} & mle & 1.3894 & 0.2991 & 1.7500 & 0 \\
\hline & mse_mle & 0.0271 & 0.0018 & & \\
\hline & mom & 1.0121 & 0.5908 & & \\
\hline & mse_mom & 0.2558 & 0.0869 & & \\
\hline & cuck:00 & 1.3689 & 0.3292 & & \\
\hline & mse_cuck 00 & 0.0364 & $8.6762 \mathrm{e}-004$ & & \\
\hline & best & cuck 00 & cuck 00 & & \\
\hline & & & & & \\
\hline \multirow{7}{*}{50} & mle & 1.4254 & 0.3277 & 1.7000 & 0 \\
\hline & mse_mle & 0.0479 & 0.0048226 & & \\
\hline & mom & 1.2055 & 0.7163 & & \\
\hline & mse_mom & 0.0937 & 0.1765 & & \\
\hline & cuck:00 & 1.3983 & 0.2991 & & \\
\hline & mse_cuck 00 & 0.0357 & $8.2262 \mathrm{e}-0.04$ & & \\
\hline & best & mle & $\mathrm{mle}$ & & \\
\hline & & & & & \\
\hline \multirow{7}{*}{100} & mle & 1.4910 & 0.3034 & 1.7877 & 0 \\
\hline & mse_mle & 0.0220 & $7.4992 \mathrm{e}-0.04$ & & \\
\hline & mom & 1.2194 & 0.6353 & & \\
\hline & mse_mom & 0.0788 & 0.1139 & & \\
\hline & cuck 00 & 1.4032 & 0.2994 & & \\
\hline & mse_cuckoo & 0.0343 & 0.0022 & & \\
\hline & best & $\mathrm{mle}$ & mle & & \\
\hline
\end{tabular}

TABLE 2: Comparison of the Different Estimators When $\beta=2$ and $\alpha=0.2$

\begin{tabular}{|c|c|c|c|c|c|}
\hline$n$ & Method & $B=2$ & $\alpha=0.2$ & $\begin{array}{c}\text { Kurtosi } \\
5\end{array}$ & Skewness \\
\hline \multirow{7}{*}{15} & mle & 1.8177 & 0.2233 & 1 & 0 \\
\hline & mse_mle & 0.0673 & 0.0017 & & \\
\hline & mom & 1.0430 & 0.6648 & & \\
\hline & mse_mom & 0.9581 & 0.2182 & & \\
\hline & cuck:00 & 1.9709 & 0.2107 & & \\
\hline & mse_cucko0 & 0.0422 & 0.0015 & & \\
\hline & best & cuck 00 & cuck 00 & & \\
\hline & & & & & \\
\hline \multirow{7}{*}{25} & mle & 1.9701 & 0.2077 & 1.7314 & 0 \\
\hline & mse_mle & 0.0407 & $8.8789 \mathrm{e}-004$ & & \\
\hline & mom & 1.1209 & 0.7815 & & \\
\hline & mse_mom & 0.7887 & 0.3383 & & \\
\hline & cuck 00 & 1.9801 & 0.2193 & & \\
\hline & mse_cuckoo & 0.0375 & 0.0016 & & \\
\hline & best & cucksoo & cucksoo & & \\
\hline & & & & & \\
\hline \multirow{7}{*}{50} & mle & 1.9914 & 0.2036 & 1.7982 & 0 \\
\hline & mse_mle & $\begin{array}{c}9.1436 \mathrm{e}- \\
004\end{array}$ & $5.7535 \mathrm{e}-004$ & & \\
\hline & mom & 1.2229 & 0.7853 & & \\
\hline & mse_mom & 0.6041 & 0.3470 & & \\
\hline & cuck:00 & 1.8305 & 0.2102 & & \\
\hline & mse_cuck 00 & 0.0425 & 0.0014 & & \\
\hline & best & mle & mle & & \\
\hline & & & & & \\
\hline \multirow{7}{*}{100} & mle & 2.0130 & 0.2198 & 1.7997 & 0 \\
\hline & mse_mle & $\begin{array}{c}7.8137 \mathrm{e}- \\
004\end{array}$ & $4.2334 \mathrm{e}-004$ & & \\
\hline & mom & 1.2277 & 0.6513 & & \\
\hline & mse_mom & 0.6014 & 0.2035 & & \\
\hline & cuck:00 & 1.9860 & 0.1971 & & \\
\hline & mse_cuck 00 & 0.0161 & 0.0012 & & \\
\hline & best & mle & mle & & \\
\hline
\end{tabular}


International Journal of Computer Science \& Information Technology (IJCSIT) Vol 9, No 5, October 2017

TABLE 3: Comparison of the Different Estimators When $\beta=2.2$ and $\alpha=0.4$

\begin{tabular}{|c|c|c|c|c|c|}
\hline$n$ & Method & $\beta=2.2$ & $\alpha=0.4$ & Kurtosis & Skemuess \\
\hline \multirow{7}{*}{15} & mle & 19464 & 0.0294 & 1.7832 & 0 \\
\hline & mse_mle & 0.1197 & 0.1129 & & \\
\hline & mom & 1.2001 & 0.7940 & & \\
\hline & mse_mom & 09998 & 0.1553 & & \\
\hline & cuckioo & 2.4615 & 03879 & & \\
\hline & mse_cuchioo & 0.8312 & 0.0031 & & \\
\hline & best & cuckion & cuckioo & & \\
\hline & & & & & \\
\hline \multirow{7}{*}{25} & mle & 19705 & 0.3929 & 1.7955 & 0 \\
\hline & mse_mle & 0.0982 & 0.0030 & & \\
\hline & mom & 1.2136 & 0.8431 & & \\
\hline & mse_mom & 09731 & 0.1968 & & \\
\hline & cuckiono & 2.1559 & 0.3919 & & \\
\hline & mse_cuckioo & 0.0480 & 0.0029 & & \\
\hline & best & cuckiono & cuckiono & & \\
\hline & & & & & \\
\hline \multirow{7}{*}{50} & mle & 2.0000 & 0.4325 & 1.7990 & 0 \\
\hline & mse_mle & 0.0804 & 0.0022 & & \\
\hline & mom & 1.2003 & 0.8501 & & \\
\hline & mse_mom & 09432 & 0.2326 & & \\
\hline & cuclioo & 2.3042 & 0.3823 & & \\
\hline & mse_cuckioo & 0.0110 & 0.0050 & & \\
\hline & best & Cuckoo & mle & & \\
\hline \multirow{7}{*}{100} & mle & 21386 & 0.4386 & 17908 & 0 \\
\hline & mse_mle & 0.0063 & 0.0021 & & \\
\hline & mom & 1.5161 & 0.7435 & & \\
\hline & mse_mom & 0.4677 & 0.1184 & & \\
\hline & Cuclioo & 2.0096 & 0.444 & & \\
\hline & mse_Cuclion & 0.0071 & 0.0022 & & \\
\hline & best & mle & mle & & \\
\hline
\end{tabular}

TABLE 4: Comparison of the Different Estimators When $\beta=3$ and $\alpha=0.33$

\begin{tabular}{|c|c|c|c|c|c|}
\hline$n$ & method & $\beta=3$ & $\alpha=0.33$ & Kurtosis & Skewness \\
\hline \multirow{7}{*}{15} & mle & 3.6985 & 0.8433 & 1.7997 & 0 \\
\hline & mse mle & 0.6205 & 0.2643 & & \\
\hline & mom & 2.1156 & 0.8854 & & \\
\hline & mse mom & 0.7822 & 0.3097 & & \\
\hline & cuckoo & 2.6793 & 0.3326 & & \\
\hline & mse_cuckoo & 0.1882 & 0.0022 & & \\
\hline & best & cuckoo & cuckoo & & \\
\hline & & & & & \\
\hline \multirow{7}{*}{25} & mle & 3.5721 & 0.6693 & 1.7990 & 0 \\
\hline & mse_mle & 0.3273 & 0.1835 & & \\
\hline & mom & 2.1598 & 0.8443 & & \\
\hline & mse_mom & 0.7059 & 0.2646 & & \\
\hline & cuckoo & 2.7307 & 0.3326 & & \\
\hline & mse_cuckoo & 0.1482 & 0.0022 & & \\
\hline & best & cuckoo & cuckoo & & \\
\hline & & & & & \\
\hline \multirow{7}{*}{50} & mle & 3.1131 & 0.3312 & 1.7945 & 0 \\
\hline & mse mle & 0.0128 & $1.4400 \mathrm{e}-006$ & & \\
\hline & mom & 2.3598 & 0.6443 & & \\
\hline & mse mom & 0.4099 & 0.0988 & & \\
\hline & cuckoo & 2.8307 & 0.3316 & & \\
\hline & mse_cuckoo & 0.0287 & $2.5600 \mathrm{e}-006$ & & \\
\hline & best & mle & mle & & \\
\hline & & & & & \\
\hline \multirow{7}{*}{100} & mle & 3.0030 & 0.3310 & 1.7800 & 0 \\
\hline & mse_mle & $\begin{array}{c}9.0000 \mathrm{e}- \\
006\end{array}$ & $1.0000 \mathrm{e}-006$ & & \\
\hline & mom & 2.5598 & 0.4443 & & \\
\hline & mse_mom & 0.1938 & 0.0131 & & \\
\hline & cuckoo & 2.9307 & 0.3313 & & \\
\hline & mse cuckoo & 0.0048 & $1.6900 \mathrm{e}-006$ & & \\
\hline & best & mle & $\mathrm{mle}$ & & \\
\hline
\end{tabular}


International Journal of Computer Science \& Information Technology (IJCSIT) Vol 9, No 5, October 2017

TABLE 5: Comparison of the Different Estimators When $\beta=1.8$ and $\alpha=0.5$

\begin{tabular}{|c|c|c|c|c|c|}
\hline$n$ & Method & $\beta=1.8$ & $\alpha=0.5$ & Kurtosis & Skewness \\
\hline \multirow{7}{*}{15} & mle & 2.4976 & 0.8870 & 1.7998 & 0 \\
\hline & mse_mle & 0.4867 & 0.1498 & & \\
\hline & mom & 1.1168 & 0.8875 & & \\
\hline & mse_mom & 0.4668 & 0.1502 & & \\
\hline & cuckoo & 1.6294 & 0.4769 & & \\
\hline & mse cuckoo & 0.0626 & 0.0046 & & \\
\hline & best & cuckoo & cuckoo & & \\
\hline & & & & & \\
\hline \multirow{7}{*}{25} & mle & 2.2176 & 0.8095 & 1.7990 & 0 \\
\hline & mse_mle & 0.1744 & 0.0959 & & \\
\hline & mom & 1.1642 & 0.8400 & & \\
\hline & mse_mom & 0.4045 & 0.1159 & & \\
\hline & cuckoo & 1.7142 & 0.4684 & & \\
\hline & mse_cuckoo & 0.0074 & 0.0052 & & \\
\hline & best & cuckoo & cuckoo & & \\
\hline & & & & & \\
\hline \multirow{7}{*}{$\mathbf{5 0}$} & mle & 1.8522 & 0.6278 & 1.7958 & 0 \\
\hline & mse mle & 0.0027 & 0.0311 & & \\
\hline & $\mathrm{mom}$ & 1.2056 & 0.7846 & & \\
\hline & mse_mom & 0.3534 & 0.0815 & & \\
\hline & cuckoo & 1.7278 & 0.4649 & & \\
\hline & mse_cuckoo & 0.0052 & 0.0057 & & \\
\hline & best & mle & cuckoo & & \\
\hline & & & & & \\
\hline \multirow{7}{*}{100} & mle & 1.8022 & 0.5349 & 1.7832 & 0 \\
\hline & mse_mle & $4.8400 \mathrm{e}-006$ & 0.0014 & & \\
\hline & mom & 1.2154 & 0.7430 & & \\
\hline & mse_mom & 0.3418 & 0.0595 & & \\
\hline & cuckoo & 1.7663 & 0.4899 & & \\
\hline & mse cuchoo & 0.0011 & 0.0039 & & \\
\hline & best & mle & mle & & \\
\hline
\end{tabular}

\section{CONClusion}

After estimating ( $\alpha$ and $\beta$ ) using the three different methods (i.e., MOM, CSO, and MLE) with different sample sizes $(n=15,25,50,100)$, we determined that the best estimator for small sample sizes $(n=15,25)$ based on MSE was the CSO estimator, as shown in Tables 1 to 5. By contrast, MLE was the best estimator for large sample sizes $(n=50,100)$. However, we conclude that the CSO estimator is the best type for small sample sizes $(n=15,25)$ because the CSO algorithm depends on the number of eggs in the host nest, which is limited.

\section{REFERENCES}

[1] Azizah Binti Mohamad, Azlan Mohd Zain \& Nor Erne Nazira Bazin, (2014), "Cuckoo Search Algorithm for Optimization Problems-A Literature Review and its Applications", Applied Artificial Intelligence An International Journal Volume 28, Issue 5.

[2] Xin She Yang and Sush Deb, "Nature \& Biologically Inspired Computing," in IEEE, University of Cambridge, Trumpinton Street, CB2 1PZ, UK, 2010.

[3] Ravi Kiran Varma P, Valli Kumari V, and Srinivas Kumar S, "A novel intelligent attribute reduction technique based on Ant Colony Optimization," International Journal of Intelligent Systems Technologies and Applicaitons, vol. 1, no. 1, pp. 23-45, 2015.

[4] Ravi Kiran Varma P, Valli Kumari V, and Srinivas Kumar S, "Feature selection using relative fuzzy entropy and ant colony optimization applied to real-time intrusion detection system," Procedia Computer Science, vol. 85, no. 2016, pp. 503-510, 2016. 
International Journal of Computer Science \& Information Technology (IJCSIT) Vol 9, No 5, October 2017

[5] Ravi Kiran Varma P, Valli Kumari V, and Srinivas Kumar S, "Application of Rough Sets and Ant Colony Optimization in feature selection for Network Intrusion Detection," International Journal of Applied Engineering Research, vol. 10, no. 22, pp. 43156-43163, 2015.

[6] Ravi Kiran Varma P, Valli Kumari V, and Srinivas Kumar S, "Ant Colony Optimization Based Anomaly Mitigation Engine," Springerplus, vol. 5, no. 1, pp. 1-32, 2016.

[7] Xin-She Yang and Suash, ""Engineering optimisation by cuckoo search"," International Journal of Mathematical Modelling and Numerical Optimisation, vol. 1, no. 4, pp. 330-343, 2010.

[8] D. S. Bunch, "Maximum Likelihood Estimation (MLE) of probabilistic choice models", SIAM Journal on Scientific and Statistical Computing, 8(1):56-70.

[9] M.S.Prasad Babu et al, (2012), "Development of Maize Expert System using Ada-Boost Algorithm and Navie Bayesian Classifier", International journal of computer Applications technology and research, volume 1-issue 3, 89-93.

[10] Persi D., "Application of the Method of Moments in Probability and Statistics", Auspices national science foundation grant DMS86-00235, Nov. 1986.

[11] Xin She Yang and Suash, "A brief literature review: Cuckoo Search and Firefly Algorithm," Studies in Computational Intelligence, vol. 516, pp. 49-62, 2014.

[12] Hongqing Zheng and Yongquan Zhou,(2013), A Cooperative Coevolutionary Cuckoo Search Algorithm for Optimization Problem", Journal of Applied Mathematics, J. Appl. Math. Volume 2013, Special Issue (2013).

[13] Najla Akram AL-Saati, Marwa Abd-AlKareem, (2013), "The Use of Cuckoo Search in Estimating the Parameters of Software Reliability Growth Models", International Journal of Computer Science and Information Security,Vol. 11, No. 6.

[14] Manjeet Kumar, Tarun Kumar Rawat,(2015), "Optimal design of FIR fractional order differentiator using cuckoo search algorithm", Expert Systems with Applications, volume 42, Issue 7, Pages 3433-3449.

[15] Prasad Babu, B.Jyothsna, (2015), "Implementation of Cuckoo Search Optimization Algorithm using Semantic Web - Based Coconut Expert System", International Journal of Advanced research in Computer Science and Software Engineering, Vol.5, Issue 9. 